\title{
Caracterización de la vulnerabilidad paisajística de un entorno natural. Aplicación en el Valle del Ambroz, municipio de Hervás
}

\section{Characterization of the landscape vulnerability in a natural environment. Exploratory study in Valle del Ambroz, Hervás municipality}

\author{
Sandra Martínez-Cuevas ${ }^{1}$, Pilar Moreno Regidor ${ }^{2}$, \\ Teresa Iturrioz Aguirre ${ }^{3}$, Daniel González Carmena ${ }^{4}$
}

\begin{abstract}
RESUMEN
Los estudios actuales de evaluación paisajística estiman la calidad y fragilidad del paisaje, empleando herramientas de análisis espacial para su valoración, caracterizando aspectos tanto ecológicos como estéticos y valorando óptimamente la vegetación como elemento crucial del paisaje. En esta línea, la presente investigación desarrolla una metodología de caracterización paisajística, diferenciando los elementos naturales y no naturales que lo conforman y valorándolos a través de dos escalas numéricas, una escala de adecuación y una escala de conservación, identificando qué elementos vulneran más o menos al paisaje y qué zonas del entorno natural deben ser remodeladas paisajísticamente. El resultado de este tipo de investigaciones puede ser utilizado por diferentes niveles de gestión pública: en primer lugar, los ayuntamientos pueden iden-
\end{abstract}

1 E.T.S. I. en Topografía, Geodesia y Cartografía. Universidad Politécnica de Madrid sandra. mcuevas@upm.es ORCID iD: http://orcid.org/0000-0002-2150-3251

2 E.T.S. I. en Topografía, Geodesia y Cartografía. Universidad Politécnica de Madrid mariapilar.moreno@upm.es ORCID iD: http://orcid.org/0000-0001-9893-5768

3 E.T.S. I. en Topografía, Geodesia y Cartografía. Universidad Politécnica de Madrid teresa. iturrioz@upm.es ORCID iD: http://orcid.org/0000-0003-2115-2719

4 E.T.S. I. en Topografía, Geodesia y Cartografía. Universidad Politécnica de Madrid daniel. glezcarmena@gmail.com ORCID iD: http://orcid.org/0000-0002-1732-0081

Copyright: (0) 2018 CSIC. Este es un artículo de acceso abierto distribuido bajo los términos de la licencia de uso y distribución Creative Commons Reconocimiento 4.0 Internacional (CC BY 4.0). 
tificar cuáles son los elementos más conflictivos que presenta el área de estudio y, en segundo lugar, las comunidades autónomas podrían incluir esta información en los Planes de Ordenación de los Recursos Naturales.

Palabras Claves: paisaje; métodos de valoración del paisaje; Sistemas de Información Geográfica; análisis raster; Valle del Ambroz.

\begin{abstract}
Current studies of landscape vulnerability estimate the quality and fragility of the landscape using spatial analysis tools for evaluation and metric analysis, characterizing ecological aspects, aesthetic and assessing the vegetation as a crucial element of the landscape. In this way, this article presents a landscape characterization methodology, analyzing both, natural and non-natural elements, and assessing them through two numeric scales, a suitability scale and a maintenance scale, identifying which elements fragment more or less the landscape and the natural environment and which areas of the natural environment should be remodeled regarding landscape. The results of this type of research can be used in different levels of governance: First, municipalities are able to identify the most conflictive elements present in the area under study; and second, from the identification of those more or less vulnerable areas, Autonomous Communities can use this information to draw up Management Plans for Natural Resources.
\end{abstract}

KEY WORDs: landscape; landscape assessment methodology; Geographical Information Systems; raster analyst; Valle del Ambroz.

Cómo citar este artículo/Citation: Martínez-Cuevas, Sandra, Moreno Regidor, Pilar, Iturrioz Aguirre, Teresa, González Carmena, Daniel (2018): "Caracterización de la vulnerabilidad paisajística de un entorno natural. Aplicación en el Valle del Ambroz, municipio de Hervás", Estudios Geográficos, LXXIX/284, pp. 61-83

\title{
INTRODUCCIÓN
}

Hervás es uno de los pueblos más representativos del norte de Cáceres, declarado Conjunto Histórico Artístico en 1969 y situado junto a la Vía de la Plata. Hervás está rodeado de paisajes abruptos con gargantas que terminan en el carismático Valle del Ambroz, territorio que se encuentra en la Red Natura 2000. Una de sus estrategias actuales dentro de la planificación turística en el Valle del Ambroz es optimizar el aprovechamiento turístico de los valores naturales y culturales, para ello se ha creado una mesa de turismo, en la que están representados los empresarios turísticos, las administraciones públicas (locales y comarcales) y DIVA (Desarrollo integral del Valle del Ambroz), para tratar la problemática asociada a la actividad turística cuyo objetivo principal 
es la oferta de turismo de naturaleza. Este trabajo de investigación se enmarca en esta línea de turismo integrado y respetuoso con la naturaleza, en la que se incluye un papel decisivo del paisaje en la creación de la imagen trasmitida al turista.

El estudio del paisaje cada vez adquiere mayor importancia en la legislación ya que, según la Ley de Patrimonio Natural y Biodiversidad, es necesario el estudio del paisaje en los Planes de Ordenación de los Recursos Naturales. Existen distintas definiciones del paisaje, las que tienen en cuenta la incidencia de las acciones de tipo natural y antrópico (Aguiló-Alonso, 2001) y las que realizan un análisis de los distintos bienes culturales que interactúan con las formas del hábitat y el aprovechamiento del medio natural (Maldonado y VelaCossio, 2011). Las transformaciones del paisaje y sus efectos medioambientales ha sido estudiada por diversos autores, Caballero Pedraza et al. (2015) analizan como en el Sureste de España la instalación masiva de invernaderos, desde un punto de vista medioambiental, manifiesta aspectos negativos: residuos generados (sólidos y líquidos), aumento de las escorrentías y por tanto de las inundaciones, o la posible contaminación del Mar.

El concepto de vulnerabilidad paisajística ha sido definido como la fragilidad o la incapacidad de los sistemas expuestos para absorber los impactos que puedan producir los fenómenos naturales o no naturales. Por esa razón, la vulnerabilidad determina la intensidad del impacto que pudiera producir la ocurrencia efectiva del riesgo sobre un territorio determinado, reflejándose en pérdidas y daños. Otero et al., (2000) tienen en cuenta, a la hora de evaluar la magnitud de la fragilidad del paisaje, los siguientes elementos: la geomorfología, la vegetación y usos del suelo, la presencia de agua visible y las infraestructuras, y Montoya et al. (2003) tienen en cuenta parámetros como el desnivel, la complejidad de formas, la diversidad y calidad visual de las formaciones, la presencia de láminas de agua, las carreteras y los núcleos urbanos.

Existen diversas metodologías para evaluar la vulnerabilidad paisajística. Un método de evaluación paisajística incorpora aspectos ecológicos y estéticos (Sancho, 1993; Daniel, 2001; Martínez Vega et al., 2003; De La Fuente et al., 2006); sin embargo, otros trabajos eliminan las valoraciones estéticas y evalúan el paisaje atendiendo exclusivamente a sus características ecológicas (Li et al., 2007). Frente a estos enfoques del paisaje desde sus características ecológicas y estéticas, existen planteamientos que evalúan su fragilidad visual (Madiedo y Bosque, 2005), definiéndola como la distancia de percepción, por el observador, de determinados elementos antrópicos del paisaje y su influencia en la valoración paisajística. En esta línea, para algunos autores, 
la valoración de la vegetación es crucial en el paisaje (Loidi, 1994; Molnár et al., 2007). Loidi (1994) realiza una cartografía en la que define las principales áreas a conservar y establece zonas prioritarias, estudiando la valoración de la vegetación a escala de paisaje. La metodología seguida en este trabajo continúa las líneas propuestas por Molnár et al., (2007) y Loidi (1994).

Algunos estudios actuales han empleado herramientas de análisis espacial para la evaluación y el análisis métrico del paisaje (Berry, 1999; McGarigal, 2002; McGarigal, 2007), caracterizando las estructuras y los cambios de ocupación y uso agroforestal. También existen aplicaciones (Ezquerra y Navarra, 2015) que estudian el paisaje visual, introduciendo variables y valorándolas en función de la cuenca visual y de la distancia al punto de observación, usando como caso de estudio las vías pecuarias. Alcántara Manzanares y Muñoz Álvarez (2015) clasifican el paisaje mediante una aplicación de métodos multivariantes clásicos en combinación con herramientas SIG, caracterizan cuatro variables perceptibles visualmente: usos del suelo, coberturas vegetales, litología y relieve, y obtienen como resultado la clasificación de ocho tipos de paisaje. Otros estudios valoran el impacto paisajístico de las construcciones realizando una estimación de la adecuación de estas al entono tomando como base una tipología de las construcciones elaborada previamente (Otero, 1996). En las últimas décadas, algunos trabajos pretenden integrar en los métodos la Ecología del Paisaje y la consideración de aspectos culturales y humanos, de manera que se combinen la ciencia de la Ecología y el arte de la Planificación del Territorio, denominada «landscape ecological aesthetic» (Thorne y Huang, 1991).

Esta investigación propone una metodología de evaluación de la vulnerabilidad paisajística que puede ser aplicada a cualquier entorno natural. El método se aplica a una zona de un gran valor paisajístico con alta frecuencia turística, el camino que une la Plaza del Convento de Hervás con el Embalse del Horcajo en el Valle de Ambroz, municipio de Hervás. Este municipio conserva en buen estado toda su parte urbana, con el famoso Barrio Judío como atractivo para los turistas. Sin embargo, la parte rústica de su entorno ha sufrido una serie de descuidos y transformaciones que demuestran una clara falta de criterio a la hora de proteger el medio natural, principal atractivo del municipio. Actualmente, dentro de las acciones concretas vinculadas al paisaje en la planificación turística, destaca por encima de todas la orientada al «embellecimiento de entornos y accesos a los recursos de interés turístico» (con más del 50\% del presupuesto dedicado a acciones de carácter paisajístico), seguida de la «implantación de rutas turísticas», «la recuperación y el tratamiento paisajístico», «la creación y recuperación de 
miradores paisajísticos», «la puesta en valor de Vías Verdes»y, finalmente, la acción dedicada al «establecimiento o mejora de la señalización turística» (Santos-Pavón et al., 2015).

Este estudio pretende dar valor al camino «Vías Verdes», recuperando y tratando el paisaje e identificando los elementos que lo vulneran. El estudio del paisaje se realiza en tres franjas visuales (próxima, intermedia y lejana), analizándose los elementos naturales y no naturales que se ven desde el camino, concretamente desde la Plaza del Convento hasta la Embalse del Horcajo. Al principio se trata la vulnerabilidad en el ámbito urbano, pero el estudio se centra principalmente en la vulnerabilidad en el medio natural. El estudio es extrapolable a otros municipios y apto para cualquier camino, carretera o senda en la que se quiera evaluar la vulnerabilidad paisajística del entorno.

Con los resultados obtenidos se pretende favorecer el desarrollo sostenible de la zona y proponer mejoras para el entorno natural ampliando la variedad de oferta para el turismo ambiental y rural, eliminando elementos vulnerables e introduciendo iniciativas que lo ayuden a desarrollarse, tanto ecológica como socialmente.

Este artículo se estructura en cuatro bloques. El primer bloque describe la metodología para caracterizar la vulnerabilidad paisajística de un entorno natural, que consiste en evaluar los elementos naturales y no naturales que vulneran el paisaje en las tres franjas visuales descritas anteriormente. En el segundo se describe la metodología aplicada en el camino que une la Plaza del Convento de Hervás con la Embalse del Horcajo. El análisis y discusión de resultados se realiza en el tercer bloque y, por último, en el bloque cuarto se presentan las conclusiones y líneas futuras de trabajo.

\section{Metodología para la CARACTERIZACión de la VUlNerabilidad PAisajística de un ENTORNO NATURAL}

El objetivo de un estudio de vulnerabilidad paisajística es la catalogación de todos los elementos, tanto naturales como no naturales, que pueden ser distinguidos y observados por el usuario en el ámbito de análisis, mediante el estudio individualizado de cada elemento y de sus atributos (Cañas, 1995). En la tabla 1 puede verse el flujo de trabajo identificando las fases principales y el desarrollo de cada una de ellas para la evaluación de la vulnerabilidad paisajística. 
TABLA 1

FLUJO DE TRABAJO PARA LA EVALUACIÓN DE LA VULNERABILIDAD PAISAJÍSTICA

EVALUACIÓN DE LA VULNERABILIDAD PAISAJÍSTICA

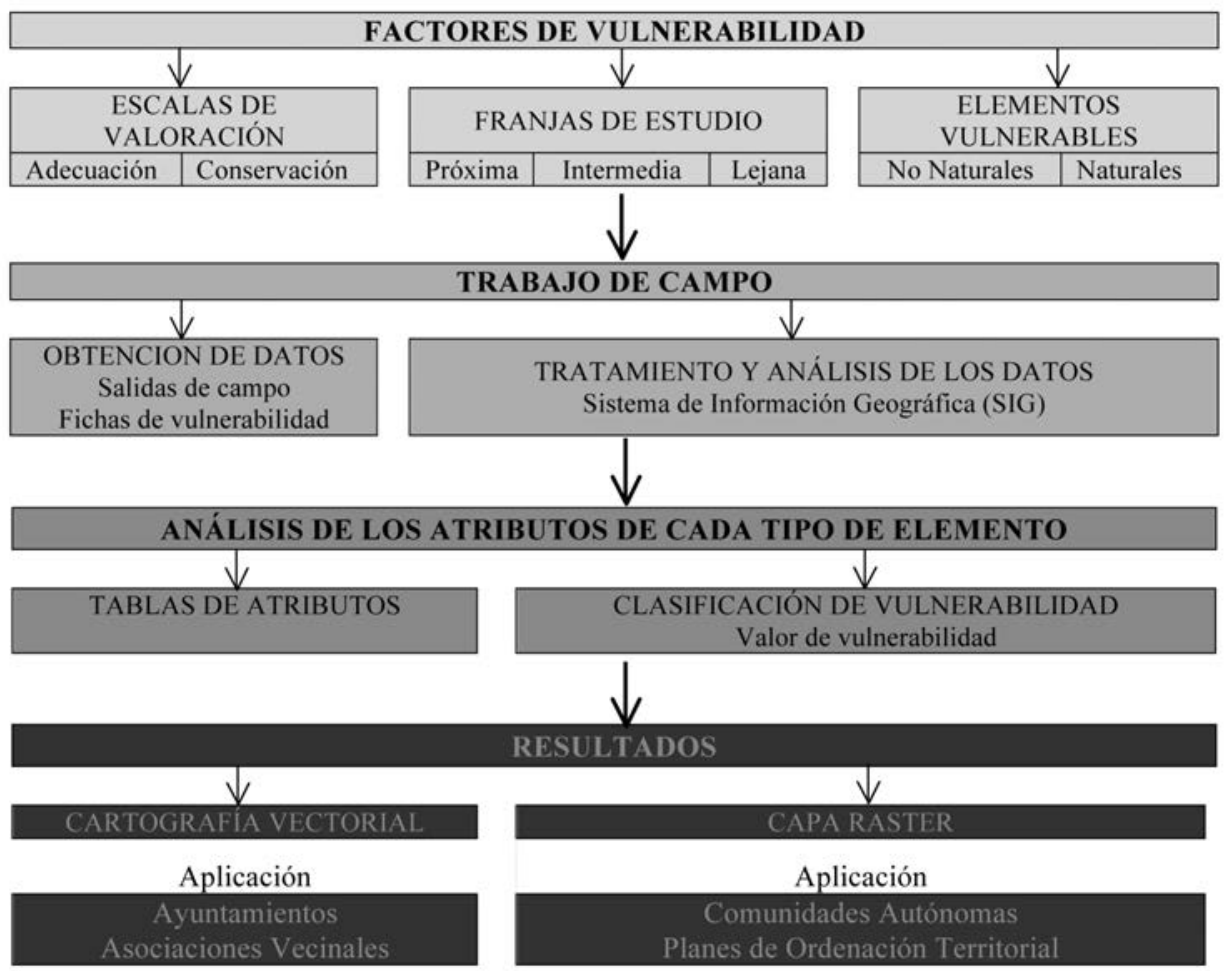

La línea metodológica se divide en tres fases. En la primera, «factores de vulnerabilidad», se identifican aquellos elementos a considerar a la hora de definir la vulnerabilidad paisajística, para posteriormente realizar un trabajo de campo donde se obtendrán los datos que se implementarán en un sistema de información geográfica (SIG, a partir de ahora). En la segunda fase, «análisis de los atributos de cada tipo de elemento», se realiza una clasificación de los elementos previamente identificados (objetos discretos) según su impacto en el paisaje. En la última fase, «resultados», se genera una superficie continua (capa raster) de vulnerabilidad paisajística, resultado de la integración de los valores de vulnerabilidad de todos los elementos estudiados. 


\subsection{Caracterización de los factores que afectan a la vulnerabilidad paisajística}

\subsubsection{Escalas de valoración}

La metodología propuesta se basa en la valoración de los elementos de la zona de estudio usando dos escalas de numéricas. Una escala de adecuación, en la que se valora si el elemento es adecuado para el entorno en el que se ubica, y una escala de conservación, con la que se valora si el elemento se encuentra en buen o mal estado. La media ponderada de las dos escalas anteriores nos daría un valor para el impacto causado por el elemento en su entorno, es decir, el grado en que vulnera el paisaje. En este estudio se considera más importante la escala de adecuación: un elemento vulnera más el paisaje por el hecho de existir en un entorno no adecuado para él, que por el mero hecho de estar mejor o peor conservado. Por tanto, para obtener el valor de la vulnerabilidad que genera cada elemento, se aplica un peso de $60 \%$ a la adecuación y de $40 \%$ a la conservación. Estas tres escalas están ordenadas de 1 a 5 (tabla 2).

TABLA 2

ESCALAS DE VALORACIÓN PAISAJÍSTICA

\begin{tabular}{c|c|c|c}
\hline & \multicolumn{3}{|c}{ ESCALAS DE VALORACIÓN PAISAJÍSTICA } \\
\hline VALOR & Adecuación & Conservación & Impacto \\
\hline 5 & muy poco adecuado & muy poco conservado & alto \\
\hline 4 & poco adecuado & poco conservado & medio \\
\hline 3 & adecuado & conservado & bajo \\
\hline 2 & muy adecuado & muy conservado & muy bajo \\
\hline 1 & óptimo & óptimo & nulo \\
\hline
\end{tabular}

\subsubsection{Franjas de estudio}

Una vez establecidas las escalas de valoración, se delimita la distancia a la que se encuentran los elementos vulnerables respecto del entorno natural a evaluar. Se han distinguido tres franjas: una franja próxima, en la que se incluye cualquier elemento que limite o se localice a corta distancia del entorno natural; una franja intermedia, formada por los elementos que están en segundo plano respecto del entorno natural hasta una distancia de unos 150 metros aproximadamente; y una franja lejana que abarca todos aquellos elementos que pueden distinguirse desde los 150 metros hasta el alcance de la vista. 
El número y tamaño de las franjas dependerá del entorno donde se realice el estudio de vulnerabilidad. En la mayoría de los casos sería conveniente establecer tres franjas, ya que permiten estructurar de forma adecuada la gran cantidad de información a gestionar, teniendo en cuenta que los criterios de valoración pueden variar considerablemente en función de la distancia a la que se encuentre el elemento analizado.

\subsubsection{Identificación de elementos de impacto}

Tras delimitar las franjas de estudio se procede a identificar los elementos que impactan en el paisaje en cada una de ellas, por ejemplo: una vivienda en la franja próxima, un cobertizo en la franja intermedia, una torre de alta tensión o un bosque de castaños en la franja lejana, etc. Una vez especificados los diferentes atributos que pudiera tener cada elemento (los materiales que forman una casa, el estado de conservación de un poste de luz, la contaminación por vertidos, etc.), hay que asignar a cada elemento una puntuación según la escala de valoración a que se refiera el atributo que se esté analizando.

En las franjas de estudio se han diferenciado dos tipos de elementos, según sean naturales o no:

a) No naturales: Aquellos elementos en los que ha intervenido el hombre para su consecución.

Se subdividen en tres categorías:

- Infraestructuras: todosloselementosqueforman partedelaredeléctrica, red de agua, mobiliario urbano, antenas de telecomunicaciones, etc.

- Edificaciones: estos elementos han sido subdivididos según su naturaleza para valorarlos de forma más exacta (residencial, terciario...).

- Caminos, trazado y derivados: son tipos de elementos exclusivos de la franja próxima.

b) Naturales: Se incluyen únicamente los dos tipos de elementos que forman el paisaje natural del valle: la vegetación y el río. Estos elementos, de alguna manera, se encuentran alterados por la acción del hombre.

Se realiza una valoración de todos los elementos según sea su estado de conservación y su grado de adecuación al entorno. Para evaluar este último factor se emplean criterios tales como el emplazamiento o los materiales, según el tipo de objeto analizado. Además, para valorar su impacto en el entorno, es necesario definir los atributos de cada componente. Dependiendo del lugar donde se realice el estudio, será necesario considerar diferentes atributos (habrá diferentes atributos que afecten a los elementos a valorar). La tabla 3 muestra los diferentes elementos naturales y no naturales identificados en cada franja de estudio. 
TABLA 3 ELEMENTOS NATURALES Y NO NATURALES IDENTIFICADOS EN LA FRANJA
PRÓXIMA, INTERMEDIA Y LEJANA
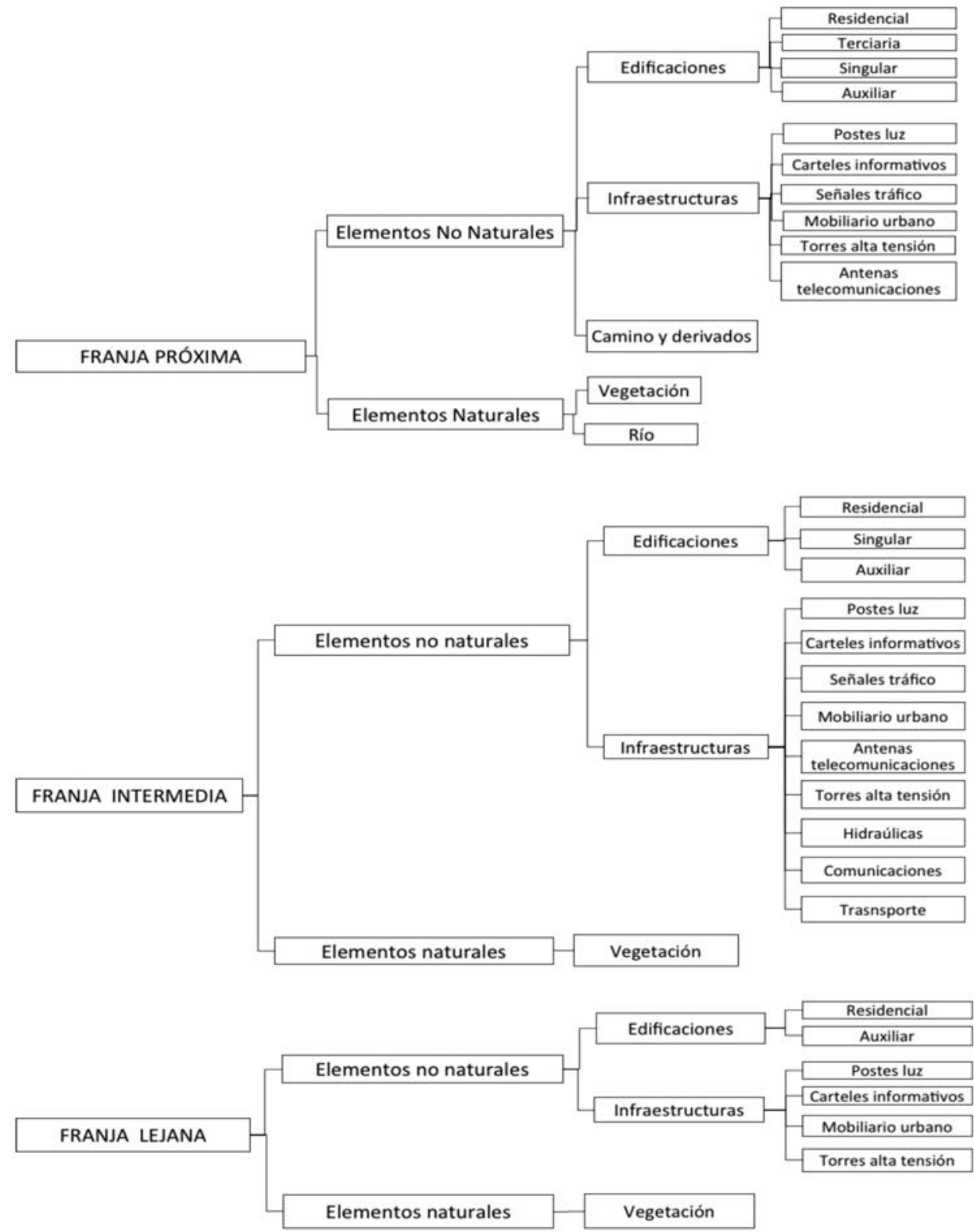

Estudios Geográficos, Vol. LXXIX, 284, pp. 61-83, enero-junio 2018 ISSN: 0014-1496, eISSN: 1988-8546, doi: https//doi.org/10.3989/estgeogr.201803 


\subsection{Obtención, tratamiento y análisis de los datos}

Una vez identificados todos los elementos y atributos necesarios para valorar la vulnerabilidad paisajística, se realiza el trabajo de campo para registrar los elementos observados en la totalidad del área de trabajo. Además de señalar la posición exacta de cada elemento a valorar, es necesaria una fotografía del mismo que, posteriormente en gabinete, servirá para puntuar de forma rigurosa sus atributos característicos.

A continuación, se integran todos los datos en un SIG, geolocalizando cada elemento y asignando un valor a cada uno de sus atributos en las diferentes escalas analizadas. Esta información permitirá, posteriormente, realizar una valoración espacial del entorno natural.

\subsection{Análisis de los atributos de cada tipo de elemento}

La valoración de un elemento se hace atributo por atributo. El criterio seguido para la puntuación de un atributo considera factores paisajísticos y urbanísticos, obtenidos a partir del contexto y conocimiento del planeamiento urbanístico local.

Como se ha explicado anteriormente, los atributos de los elementos se valorarán con una puntuación de 1 a 5, siendo " 1 " el valor óptimo (no hay impacto y por tanto, no se vulnera el paisaje) y "5" la peor valoración, cuando el paisaje se vulnera muy negativamente. En los análisis de los elementos naturales y no naturales identificados, se valoran todos los atributos del elemento que pertenezcan a las escalas de adecuación y de conservación. El valor de impacto del elemento se obtiene con la ecuación 1, que sería la suma ponderada de los valores medios de adecuación y conservación de cada atributo, aplicando un peso del $60 \%$ y $40 \%$ respectivamente.

donde:

$$
V I_{e}=0.6 \frac{\sum V_{a}}{N_{a}}+0.4 \frac{\sum V_{c}}{N_{a}}
$$

$V I_{e}=$ valor de impacto del elemento; $V_{a}=$ valor de adecuación para ese elemento $V_{c}=$ valor de conservación para ese elemento; $N_{a}=$ número de atributos de la escalá de valoración.

Para homogeneizar los resultados del análisis, todos los elementos de la misma categoría o grupo (edificaciones, infraestructuras, etc.) se deben caracterizar con los mismos criterios. Así, una vivienda unifamiliar se valoraría con los mismos atributos empleados para valorar un edificio colectivo de 
TABLA 4

\section{ATRIBUTOS CONSIDERADOS EN LAS ESCALAS DE VALORACIÓN DE LOS DIFERENTES ELEMENTOS EN LAS FRANJAS DE ESTUDIO}

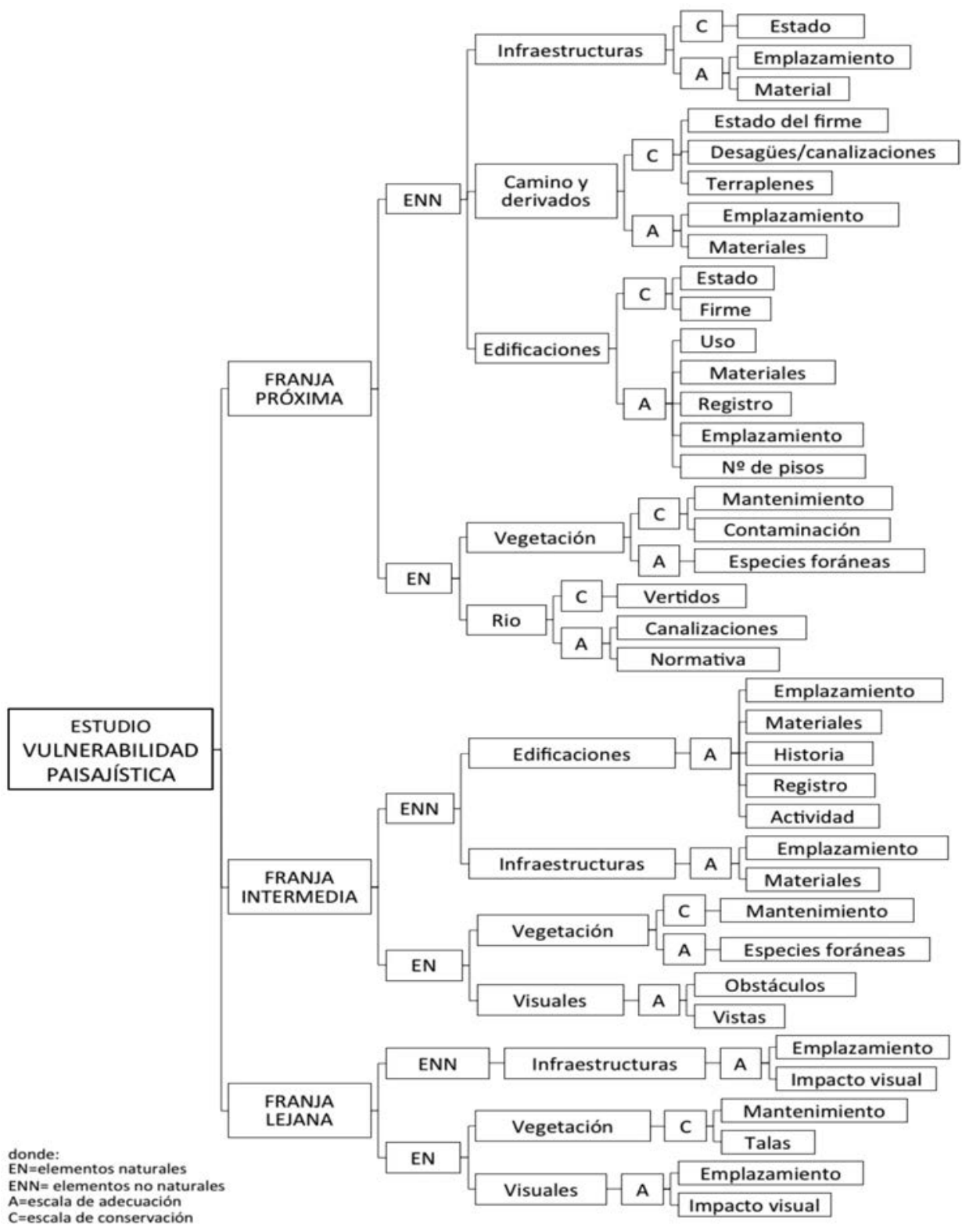

Estudios Geográficos, Vol. LXXIX, 284, pp. 61-83, enero-junio 2018 ISSN: 0014-1496, eISSN: 1988-8546, doi: https//doi.org/10.3989/estgeogr.201803 
viviendas en función de la franja de estudio donde se encuentre. Por ejemplo, en la franja próxima para la escala de conservación, se caracterizarán su estado y firme y, para la escala de adecuación, el uso, material, registro, emplazamiento y número de pisos. Sin embargo, los criterios a seguir para caracterizar un cartel informativo, situado también en la franja próxima, serán bien diferentes, de modo que para la escala de conservación se considerará su estado y para la escala de adecuación el material, registro y el emplazamiento. En la tabla 4 se describen los atributos que intervienen en la valoración de los diferentes elementos en las franjas de estudio.

\subsection{Análisis y presentación de resultados}

Con este tipo de estudio se pueden identificar los elementos que vulneran más o menos al paisaje, así como aquellas zonas del entorno natural que deben ser remodeladas paisajísticamente por la mala adecuación y conservación de sus elementos. El resultado del análisis es una superficie o capa raster de la zona representado por medio de una simbología superficial basada en claroscuros. Estas gamas de color asocian colores claros con valores bajos de vulnerabilidad, de forma que los colores se van oscureciendo según aumentan dichos valores.

Este modelo presenta una doble aplicación. Por un lado permite que los ayuntamientos puedan identificar los elementos más conflictivos en el área de estudio, así como establecer jerarquías entre los mismos según la importancia de las deficiencias a corregir. Por otro lado, la representación del entorno natural, con la indicación de las zonas más o menos vulnerables paisajísticamente y convenientemente jerarquizadas, puede ser usada por las comunidades autónomas para redactar los nuevos Planes de Ordenación Territorial y los Planes de Ordenación de los Recursos Naturales.

Estos resultados, junto con el conocimiento de los diferentes factores que forman el conglomerado local, es decir, el contexto histórico, cultural y social del entorno, permitirán diseñar iniciativas adecuadas para favorecer el desarrollo local del área e impedir futuras agresiones al medioambiente.

2. Ambito de actuación: camino que une la población de Hervás y el Embalse DEL HORCAJO

Esta metodología se aplica a una zona de un alto valor paisajístico y elevada frecuencia de uso, el camino que une la Plaza del Convento de Hervás con la Embalse del Horcajo en el Valle de Ambroz, municipio de Hervás. En la figura 1, 
FIGURA 1

ORTOFOTOGRAFÍAS DEL ÁMBITO DE ACTUACIÓN

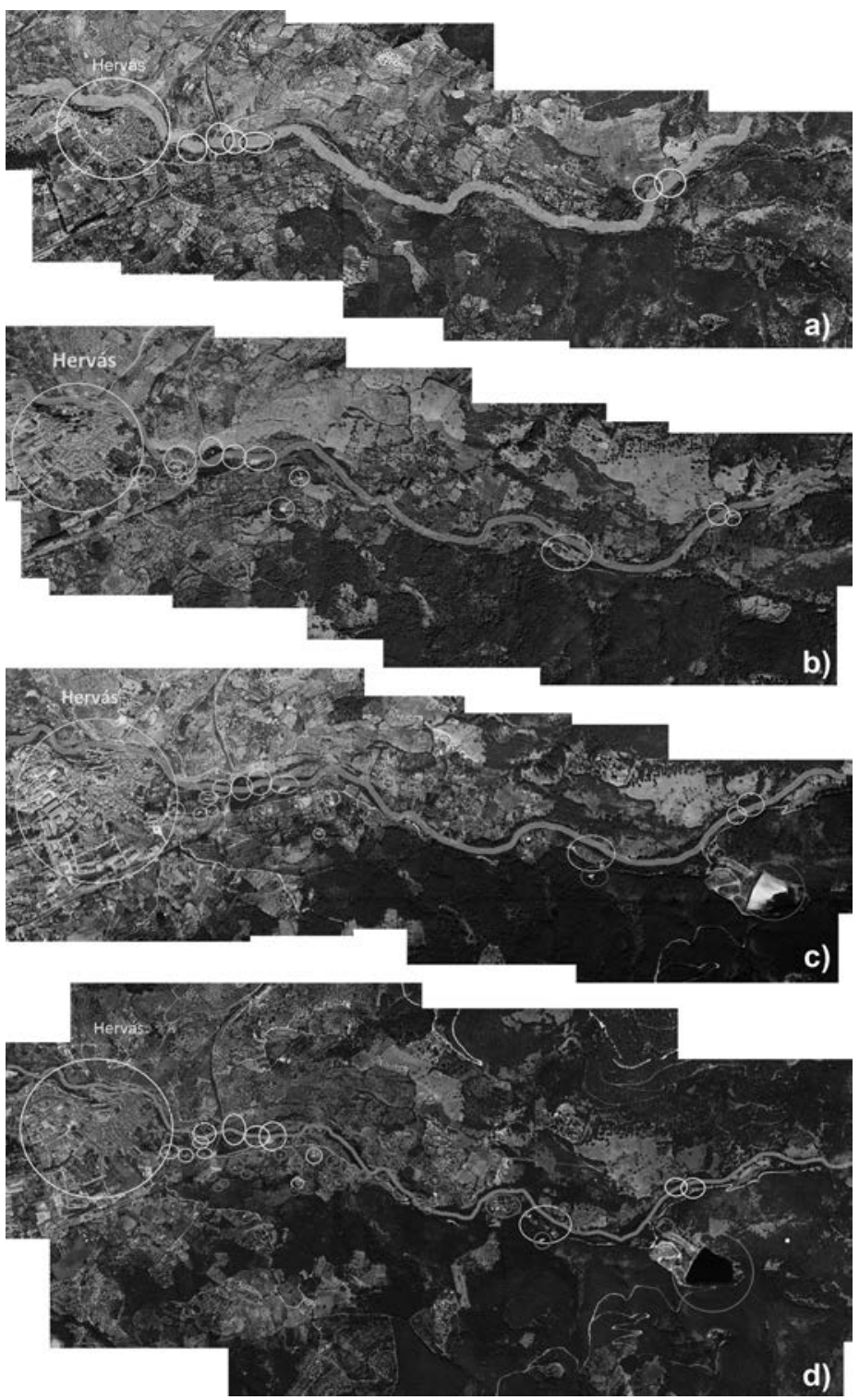

a) de 1956 (en amarillo se han rodeado las edificaciones existente), b) de 1986 (en verde se han rodeado las nuevas edificaciones respecto a 1956), c) de 2001 (en rosa se han rodeado las nuevas edificaciones respecto a 1986) y d) de 2009 (en rojo se han rodeado las nuevas edificaciones respecto a 2009). 
se muestran mosaicos de ortofotografías de alta resolución del camino de estudio y su entorno, procedentes del Instituto Geográfico Nacional (IGN) y tomadas en 1956, 1985, 2001 y 2007. En estas imágenes puede observarse la gran cantidad de edificaciones que se han ido construyendo a lo largo de los años, lo que evidencia una ausencia total de control y criterio medioambiental y paisajístico.

En la figura la) de 1956, se indican las escasas edificaciones existentes más allá de la Plaza del Convento y alejadas del casco urbano. La figura 1b) de 1986 muestra, las nuevas edificaciones respecto de las de 1956. Como novedad, aparece alguna construcción aislada al sur del río Ambroz, en el tramo situado entre la Plaza del Convento y el Puente del Batán, así como el depósito de agua, que es la edificación más alejada, hacia el sur, respecto del camino de estudio y del río. La figura 1c) de 2001 muestra, las nuevas edificaciones respecto de las de 1986. Con el incremento del turismo y el desarrollo de los municipios, comienza el aumento descontrolado de las edificaciones a lo largo del valle y el inicio de la agresión urbanística al Valle del Ambroz. Muchas de las nuevas edificaciones son del tipo vivienda y otras del tipo auxiliar (cobertizos, cabañas) que más tarde evolucionarán a viviendas. La figura 1d) de 2009 muestra, en rojo, las nuevas edificaciones respecto de las de 2001. En esta imagen es patente el descontrol constructivo, ya originado en los años 80 , que se prolonga dando lugar a nuevos elementos que impactan de forma negativa en el paisaje.

\subsection{Clasificación de los elementos según su impacto visual}

En el camino se han delimitado tres franjas de estudio con las dimensiones indicadas en el apartado 2.2.2 de la metodología (figura 2).

El estudio caracteriza los atributos de cada uno de los elementos que se ven desde el camino en cada una de las franjas, todo ello desde la Plaza del Convento hasta la Embalse del Horcajo, ya que el camino continúa, montaña arriba, hacia la central hidroeléctrica de «La Luz»y hacia «Las Chorreras».

Se enumeran los diferentes grupos de elementos que se pueden observar desde el camino en cada una de las franjas, por ejemplo: una vivienda en la franja próxima, un cobertizo en la franja intermedia, bien una torre de alta tensión en la franja lejana, la ribera de un río en la franja próxima o un bosque de castaños en la franja lejana, según puedan observarse con cualquier visor cartográfico, por ejemplo Google Earth o el SIGPAC, Sistema de Información 


\section{FIGURA 2}

FRANJAS DE ESTUDIO: PRÓXIMA, INTERMEDIA Y LEJANA

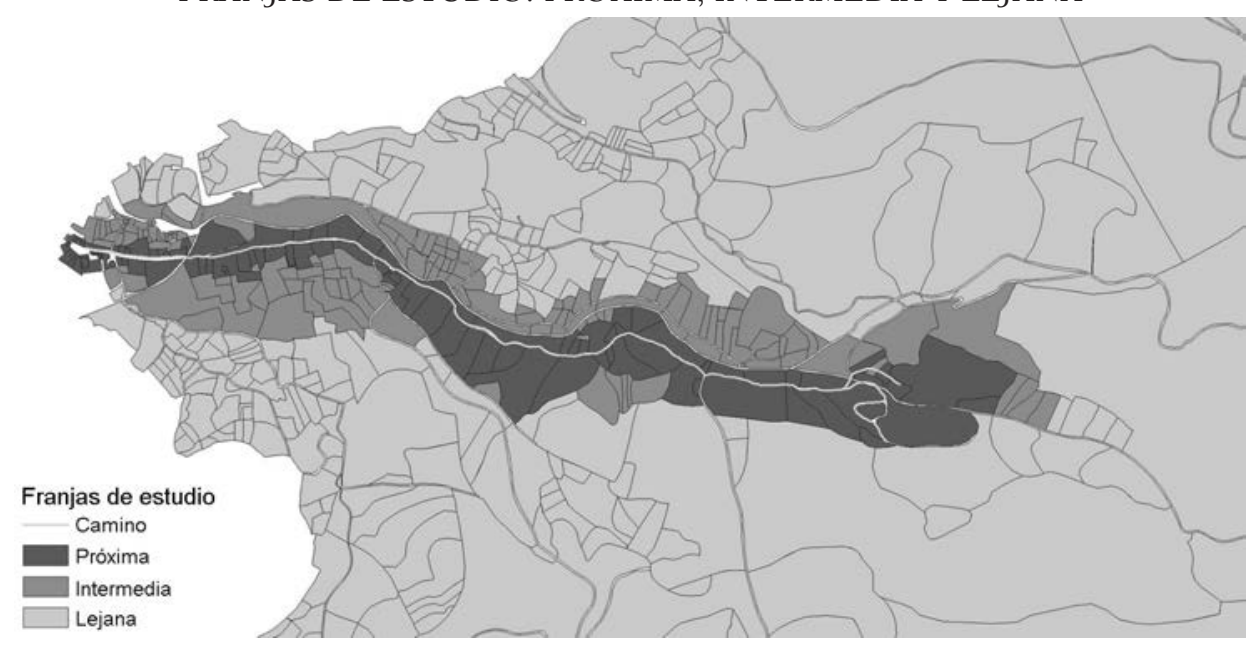

Geográfica de Parcelas Agrícolas, del MAGRAMA. En la figura 3 se muestra una infraestructura de un cartel informativo bien conservado (figura 3a), que tendrá un valor óptimo (1) para la escala de conservación, y un cartel mal conservado (figura 3b), que tendrá, para esta misma escala, un valor pésimo (5). En la (figura 3c), se muestra un ejemplo de edificación mal adecuada al entorno natural, al que se asigna un valor de 5, y una edificación adecuada (figura 3d), con un valor de 1 .

A partir de lo anterior se realiza la clasificación de los diferentes atributos que pudiera tener cada elemento: los materiales que forman una casa, el estado de conservación de un poste de luz, la contaminación por vertidos en el camino, etc. Hay que tener en cuenta que el escenario de estudio puede ser totalmente dispar y, por lo tanto, los elementos que aparecen pueden variar.

Los valores de impacto para el elemento se calculan mediante una suma ponderada de sus valores medios de adecuación y de conservación, ecuación (1) del apartado 2.3, dando un peso mayor a la adecuación que a la conservación, de $60 \%$ y $40 \%$ respectivamente.

En la figura 4 se representan, en un tramo del camino, los edificios e infraestructuras clasificados según la vulnerabilidad que generan en el entorno. Lo mismo se ha hecho para la vegetación y río. 
FIGURA 3

\section{INFRAESTRUCTURAS DE LA ZONA DE ESTUDIO}
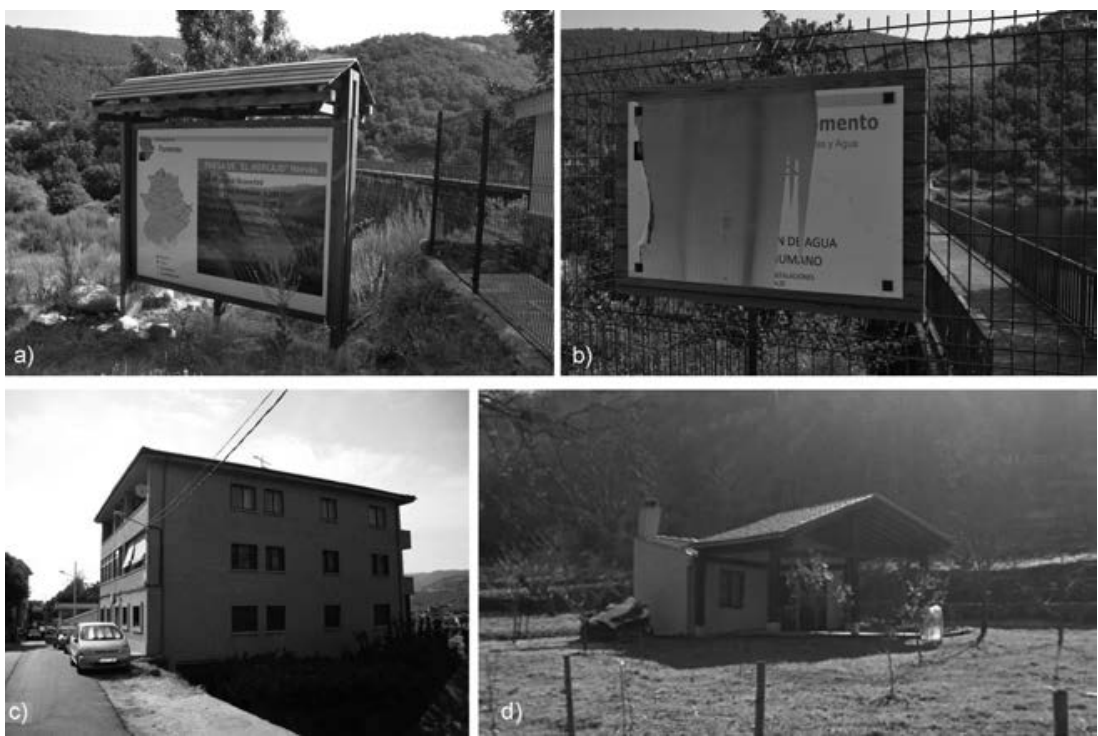

a) Ejemplo de infraestructura en buen estado de conservación, b) ejemplo de infraestructura en mal estado de conservación, c) ejemplo de edificación poco adecuada para el entorno natural y d) edificación adecuada.

\section{FIGURA 4}

VULNERABILIDAD GENERADA EN EL PAISAJE POR LAS INFRAESTRUCTURAS Y EDIFICACIONES EXISTENTES EN LAS TRES FRANJAS DE ESTUDIO.

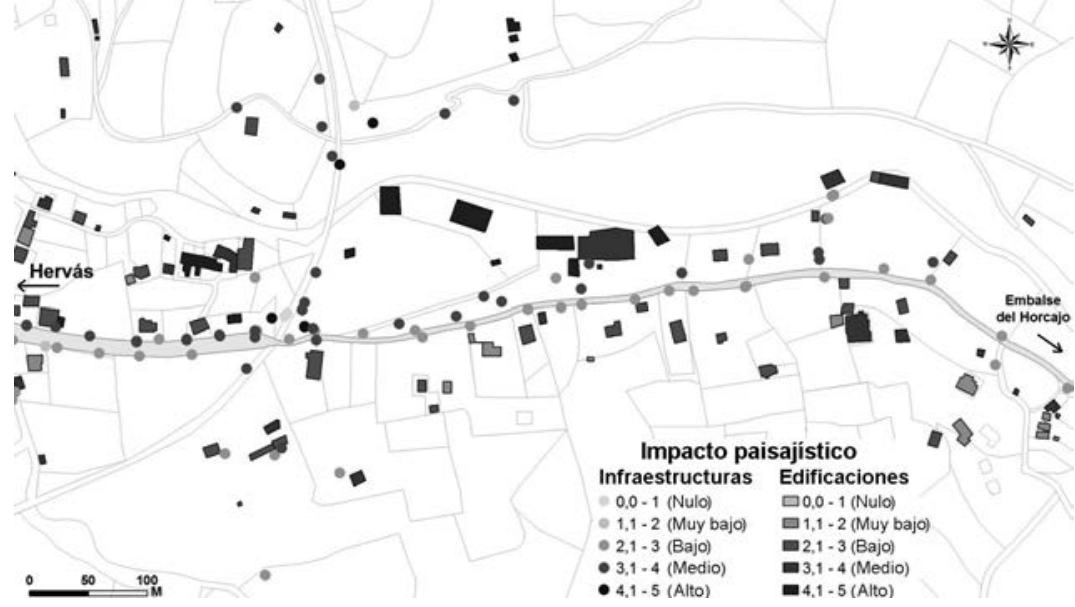

Estudios Geográficos, Vol. LXXIX, 284, pp. 61-83, enero-junio 2018

ISSN: 0014-1496, eISSN: 1988-8546, doi: https//doi.org/10.3989/estgeogr.201803 


\subsection{Método raster de obtención de la capa de vulnerabilidad}

A partir de los valores calculados anteriormente, se ha creado una capa raster que representa los datos de la variable vulnerabilidad paisajística, cuyo valor en cada punto se ha obtenido a partir de los valores correspondientes al impacto causado por las edificaciones, infraestructuras, camino, río y vegetación. Para ello se han utilizado varios métodos, de los que se describe el de mejores resultados:

- En una primera aproximación, la vulnerabilidad de la zona de estudio se calcula como el resultado de la amenaza generada por las edificaciones e infraestructuras existentes. En los puntos del espacio donde no existan estos elementos, el valor asignado será la suma ponderada de los valores del impacto causado por dichos elementos, usando como factor de ponderación el inverso de la distancia a ellos (o el inverso de la distancia elevado a una cierta potencia). Se obtiene la capa EI (Edificaciones e Infraestructuras).

- Se considera que la vegetación mejora los valores de impacto causados por las edificaciones e infraestructuras (capa EI). La mejora implica que estos últimos valores han de disminuir, teniendo en cuenta que 1 representa un valor óptimo (no se vulnera) y 5 un valor pésimo (vulnera). El criterio a implementar se muestra en la tabla 5. Los puntos del terreno donde se desconozca el valor de mejora debido a la vegetación, tendrán por valor el impacto ya calculado en la capa EI. Se genera la capa VEI (Vegetación, Edificaciones e Infraestructuras).

TABLA 5

MEJORA DEL IMPACTO CAUSADO POR EDIFICACIONES E INFRAESTRUCTURAS DEBIDO A LA VEGETACIÓN

\begin{tabular}{c|c}
\hline Valoración de la vegetación & $\begin{array}{c}\text { mejora del impacto causado por } \\
\text { edificaciones } \text { e infraestructuras }\end{array}$ \\
\hline 1 & $20 \%$ \\
\hline 2 & $20 \%$ \\
\hline 3 & $10 \%$ \\
\hline 4 & $10 \%$ \\
\hline 5 & $0 \%$ \\
\hline
\end{tabular}


La vulnerabilidad del paisaje debida al camino y al río, en los puntos del espacio ocupados por estos elementos, dadas sus características y relevancia, se calcula como la suma ponderada de sus valores y de los correspondientes valores de impacto de las edificaciones, infraestructuras y vegetación de la zona de estudio (capa VEI). Los factores de ponderación pueden variarse, teniendo siempre en cuenta la prioridad y mayor representatividad del camino y del río:

$$
\begin{gathered}
\text { VulnerabilidadFinal_camino/río }= \\
\text { Impacto_camino/río }{ }^{*} 0,8+\text { Impacto_Edif_Infr_Veg }{ }^{*} 0,2
\end{gathered}
$$

De esta forma, el resultado de la vulnerabilidad debida al camino y al río se ve afectado por el impacto de los otros elementos y no al revés. Con estos datos se generará una capa raster $(\mathrm{C} 2)$ con valores en las posiciones correspondientes al camino y río.

La capa de vulnerabilidad del paisaje se obtiene a partir de las capas VEI y $\mathrm{C} 2$, con los siguientes criterios:

- En los puntos del espacio ocupados por el camino y el río, la vulnerabilidad será la calculada en la capa C2.

- En el resto de puntos del espacio de la zona de estudio, la vulnerabilidad será la calculada en la capa VEI.

En la figura 5 puede verse la capa final raster de vulnerabilidad paisajística del camino y su entorno.

\section{FIGURA 5}

\section{CAPA RASTER DE VULNERABILIDAD PAISAJÍSTICA DEL CAMINO Y SU ENTORNO}

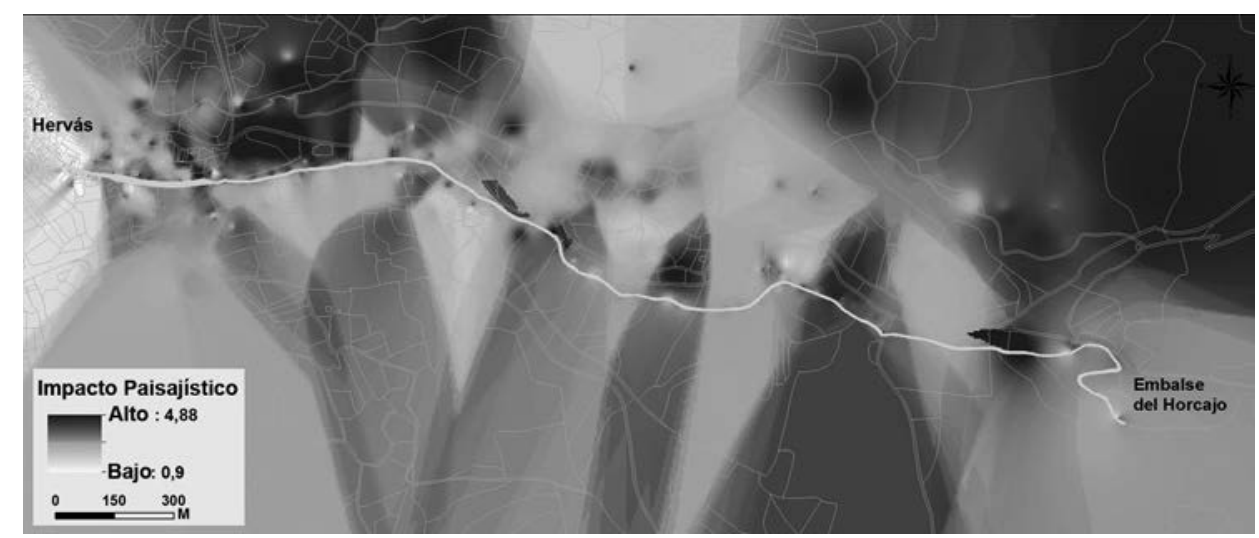

Estudios Geográficos, Vol. LXXIX, 284, pp. 61-83, enero-junio 2018

ISSN: 0014-1496, eISSN: 1988-8546, doi: https//doi.org/10.3989/estgeogr.201803 


\section{RESUlTADOS Y DisCUSIÓN}

De la aplicación que se presenta en este artículo se derivan una serie de discusiones referentes al algoritmo de interpolación, los pesos asignados a las dos clases de elementos (naturales y no naturales) y a la influencia de la vegetación en el cálculo de los valores del impacto paisajístico.

La primera discusión que plantea esta investigación es conocer cuál de los dos métodos de interpolación aplicados para generar la capa de vulnerabilidad, debida a las infraestructuras y edificaciones, sería el más adecuado en este tipo de análisis: el inverso de la distancia al cuadrado o el inverso de la distancia al cubo. Tal y como puede observarse en la figura 6 , cuando se aplica el inverso de la distancia al cubo aumentan considerablemente los valores de vulnerabilidad, sobre todo en las zonas más alejadas del camino.

\section{FIGURA 6}

\section{MÉTODOS DE INTERPOLACIÓN}

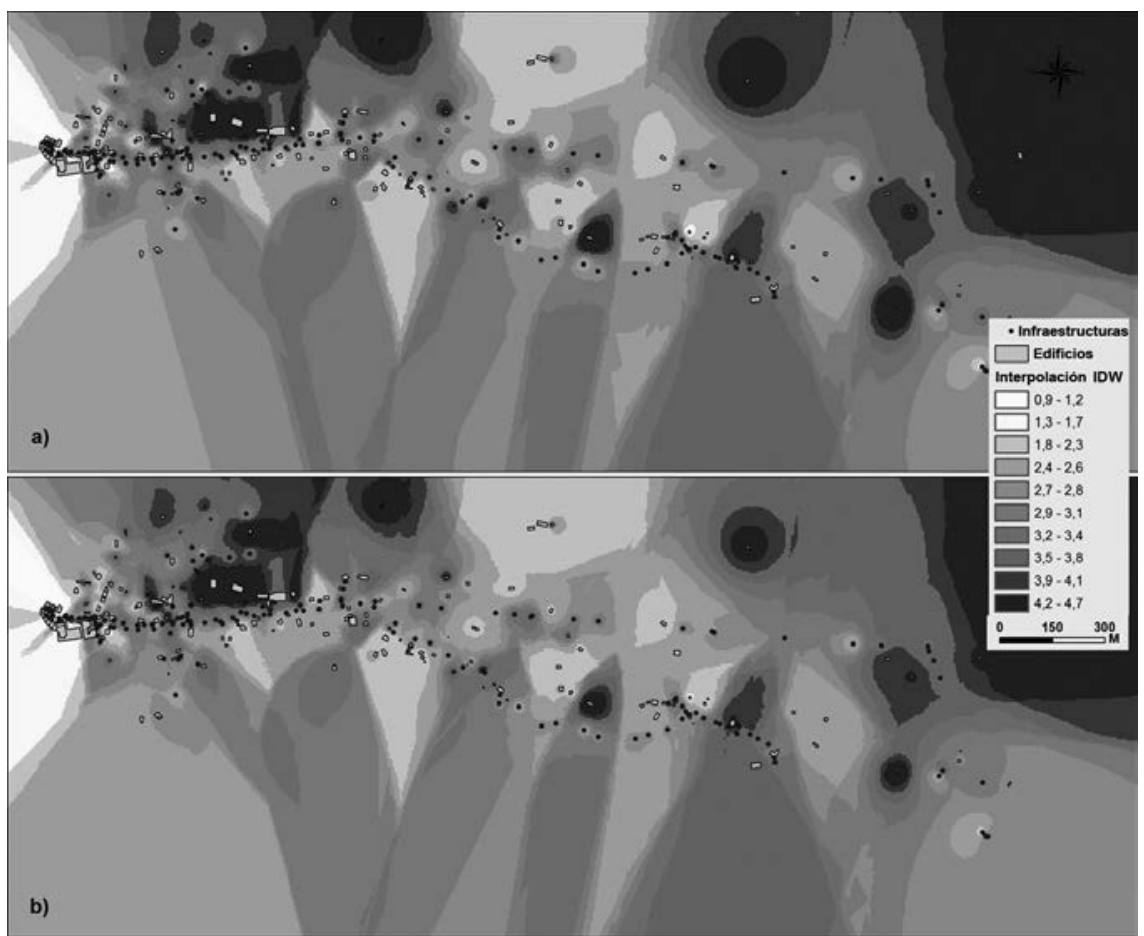

a) aplicación de la herramienta idw2 y b) idw3 para generar la capa de vulnerabilidad debida a infraestructuras y edificaciones. 
Teniendo en cuenta que la influencia de los objetos disminuye con la distancia y que, a mayor potencia, esta influencia se atenúa más rápidamente, en este caso se cree conveniente utilizar el inverso de la distancia al cuadrado, si bien el estudio se ha realizado usando las dos potencias para comparar los resultados finales.

Además, también se realizaron diferentes pruebas con los pesos asignados a la vulnerabilidad causada por los elementos Camino/Río e Infraestructuras/ Edificaciones. En el resultado final se ha aplicado una ponderación del $80 \%$ a la capa Camino/Río y del 20\% a la capa Edificaciones/Infraestructuras, al considerar que la valoración de los aspectos ecológicos (río) debe tener un mayor peso en la vulnerabilidad del paisaje (10) y que una buena adecuación y conservación de estos elementos es crucial para su valoración en este entorno natural.

En cuanto a la influencia positiva de la vegetación en el cálculo del impacto, se obtienen resultados no realistas cuando esta se pondera con valores muy altos y es necesario ajustar su peso considerando otros factores, siempre en el sentido de mejora del valor final. De acuerdo a la tabla 5, las valoraciones 1 y 2 aumentan en un 20\% el valor del impacto final, al tratarse de especies autóctonas y con buen mantenimiento. Cuando la vegetación es foráneas y presenta talas se asignan los valores 3 y 4 , que aunque el mejoran el resultado final solo lo hacen en un $10 \%$.

Los mayores valores de vulnerabilidad paisajística se dan en la franja cercana al camino, donde las infraestructuras son poco adecuadas, sobre todo por su emplazamiento y materiales. Además, el número de edificaciones abandonadas y en ruinas es elevado, como el grupo de antiguas fábricas textiles que se encuentran en un lugar muy expuesto y despejado, junto al Puente de Hierro.

\section{CONCLUSIONES}

En este trabajo se describe un método de caracterización de la vulnerabilidad paisajística de un entorno natural, aplicado al camino que une Hervás con el Embalse del Horcajo, siendo un modelo fácilmente extrapolable a cualquier otro ámbito, ya sea urbano, rural o mixto. Con los resultados obtenidos se puede visualizar de manera rápida aquellas zonas donde existe una mayor vulnerabilidad paisajística y de las que, por tanto, sea necesario realizar un estudio de mejora.

La metodología desarrollada, en la franja próxima e intermedia, caracteriza los elementos que vulneran el paisaje de una forma precisa, con un resultado riguroso y de gran calidad (especialmente en la franja próxima). Por tanto, esta metodología podría sustituir (en la franja próxima) o complementar (en las franjas intermedia y lejana) el tipo de análisis comúnmente desarrollado en otros 
trabajos de investigación, donde la fragilidad se evalúa atendiendo a criterios como la pendiente y orientación del terreno, la altura y densidad forestal, cuencas visuales, etc. Además, este tipo de metodología podría enriquecerse con la planificación colaborativa espacial. Por ejemplo, se podrían implementar iniciativas de trabajo colaborativo turístico para la integración de construcciones turístico rurales, basadas en el intercambio de información y la colaboración entre los agentes implicados en la zona de estudio (Jeong et al., 2014).

De la aplicación de la metodología al entorno natural de Hervás se concluye que, en esta zona, es posible abordar medidas concretas para mejorar la calidad turística y paisajística tales como: la restauración y rehabilitación de las antiguas fábricas textiles situadas cerca del Puente de Hierro y del Puente del Batán, transformándolas en centros de visita e interpretación, lo que aumentaría el valor paisajístico de la zona y supondría un atractivo más para el turismo rural del pueblo; la restauración del puente de la antigua línea de ferrocarril abandonada y de la misma línea de ferrocarril, y la recuperación de la antigua piscifactoría abandonada. Otros aspectos a valorar serían: las deficientes condiciones de conservación en las que se encuentra el Embalse del Horcajo; la cantidad de contaminación de desechos plásticos y de diferente índole situada en los aledaños de la rivera del Ambroz y en el mismo río; las malas condiciones de ciertos terraplenes a los lados del camino, etc. En conclusión, se evidencia un grave incumplimiento de normas y criterios relacionados con el medio natural en el valle.

En este tipo de investigaciones queda plasmada la elevada capacidad de los SIG para estudiar y gestionar problemas paisajísticos y medioambientales. Además, la clasificación de los elementos de la zona estudiada permite tener un conocimiento más completo de la misma. Se concluye, de los resultados obtenidos, que la metodología idónea para calcular la vulnerabilidad paisajística de una zona es aquella en la que los valores del impacto causado por los elementos se ven mejorados al introducir en el estudio, y en los cálculos de impacto, la variable vegetación.

\section{REFERENCIAS}

Aguiló-Alonso, M. (2001). "Guía Metodológica para estudios del Medio Físico”, Madrid: Ministerio de Medio Ambiente.

Alcántara Manzanares, J. y Muñoz Álvarez, J.M. (2015). "Clasificación del paisaje de Huelva (España): Un método objetivo de identificación y caracterización" Estudios Geográficos, Vol. LXXVI, 279, pp. 447-471, julio-diciembre 2015 ISSN: 0014-1496, eISSN: 1988-8546, doi: 10.3989/estgeogr.201516 
Berry, J., Buckley, D. J., y McGarigal, K. (1999). "Integrating Landscape Structure Programs with GIS". <www.innovativegis.com>.

Caballero Pedraza, A.; Romero Díaz, A. y Espinosa Soto, I. (2015). "Cambios paisajísticos y efectos medioambientales debidos a la agricultura intensiva en la Comarca de Campo de Cartagena-Mar Menor (Murcia)" Estudios Geográficos, Vol. LXXVI, 279, pp. 473-498, julio-diciembre 2015, ISSN: 0014-1496, eISSN: 1988-8546, doi: 10.3989/estgeogr.201517

Cañas, I. (1995). "Valoración del paisaje". Lugo: Unicopia.

Daniel, T.C. (2001). "Whither scenic beauty? Visual landscape quality assessment in the 21st century", Landscape and Urban Planning. 54: 267-281.

De La Fuente, G., Atauri, J.A. y De Lucio, J. V. (2006). "Relationship between landscape visual attributes and spatial pattern indices: a test study in Mediterranean climate landscapes", Landscape and Urban Planning. 77: 393-407.

Ezquerra, A., Navarra, M. (2015). "Metodología de valoración de paisaje empleando ArcGIS: Aplicación a las Vías Pecuarias", Informes de la Construcción 67(537): eo66, doi: http: //dx. doi.org/ 10.3989 /ic.13.051.

Instituto Geográfico Nacional. http//: www.ign.es

Jeong, J.S., García-Moruno, L., Hernández-Blanco, J. (2014). "Un modelo web para la asistencia en la toma de decisiones en la integración de las construcciones rurales mediante planificación espacial multicriterio", Informes de la Construcción, 66(533):e004, doi:http://dx.doi.org/10.3989/ic.13.001.

Li, X., Xiao, D., He, X., Chen, W. y Song, D. (2007). "Evaluation of landscape changes and ecological degradation by GIS in arid regions: a case study of the terminal oasis of the Shiyang River, northwest China", Environmental Geology. 52 (5): 947-956.

Loidi, J., (1994). "Phytosociology applied to nature conservation and land management", En Song, Y., Dierschke, H. y Wang, X. (Eds.). Applied Vegetation Ecology. 35th Sympo- sium IAVS in Shangai. East China Normal Univ. Press.

Madiedo, F. y Bosque, J. (2005). "Una propuesta metodológica para cuantificar la visibilidad del territorio por la población”, En Seguinot, J. (Ed.). Las Tecnologías Geográficas. Aplicaciones locales para la conservación global, San Juan de Puerto Rico, Universidad de Puerto Rico, 12p.

Maldonado, L., Vela-Cossio, F. (2011). "El patrimonio arquitectónico construido con tierra. Las aportaciones historiográficas y el reconocimiento de sus valores en el contexto de la arquitectura popular española", Informes de la Construcción, 63(523): 71-80, doi: http://dx.doi.org/10.3983/ic.10.062

Martínez Vega, J., Martín, M. P. y Romero Calcerrada, R. (2003). "Valoración del paisaje en la Zona de Especial Protección de Aves Carrizales y Sotos de Aranjuez (Comunidad de Madrid)", GeoFocus. 3: 1-21

MCGarigal, K., Marks, B., Holmes, C. y Ene, E. (2002). "Fragstats 3.3. Spatial Pattern Analysis Program for Quantifying Landscape Structure", Department of Natural Resources Conservation. University of Massachusetts. 
McGarigal, K., Ene, E. y Holmes, C. (2007). "Spatial Pattern Analysis Program for Categorical Maps", University of Massachusetts.

Molnár, Z., Bartha, S., Seregélyes, T., Illyés, E., Botta-Dukát, Z., Timár, G., Horváth, F., Révész, A., Kun, A., Bölöni, J., Biró, M., Bodonczi, L., Deák, A., Fogarasi, P., Horváth, A., Isépy, I., Karas, L., Kecskés, F., Molnár, C., Ortmann, A. y Rév, S. (2007). "A grid-based, satellite image supported, multiattributed vegetation mapping method (MÉTA)", Folia Geobotanica. 42 (3): 225-247.

Montoya Ayala, R.; Padilla Ramírez, J.; Standford Camargo, S. (2003). "Valoración de la calidad y fragilidad del paisaje en el Valle de Zapotitlán de las Salinas, Puebla (Mexico)", Boletín de la A.G.E. 35; p123-136.

Otero, I., Novoa, J. C. y Hernández, M. (1996). "Valoración del paisaje y del impacto paisajístico de las construcciones en el Páramo Leones", Informes de la Construcción, Vol. 47, pp. 115-136

Otero, C., Togores, R., De La Pedraja, A., Bruschi, V., González, A. (2000). "Métodos gráficos en la modelización, simulación y evaluación del impacto ambiental», XII Congreso Internacional de Ingeniería Gráfica. Valladolid. June 2000.

Sancho, J., Bosque, J. y Moreno, F., (1993). "Crisis and permanence of the traditional Mediterranean landscape in the central region of Spain", Landscape and Urban Planning. 23:155-166.

Santos-Pavón, E; Fernández-Tabale, A; y Muñoz-Yule, O. (2015) "La incorporación del paisaje a la planificación turística. Análisis de la estrategia de turismo sostenible de Andalucía”, Cuadernos de Turismo, no 37, (2016); pp. 175-202 Universidad de Murcia, ISSN:1139-7861 e ISSN:1989-4635 DOI: http://dx.doi.org/10.6018/turismo.37.256201

Thorne, J. F. y Huang, C.-S. (1991). "Toward a landscape ecological aesthetic: methodologies for designers and planners", Landscape and Urban Planning. 21: 61-79.

Fecha de recepción: 15 de julio de 2016.

Fecha de aceptación: 6 de noviembre de 2017. 